\title{
The impact of disinvestment on alcohol and drug treatment delivery and outcomes: a systematic review
}

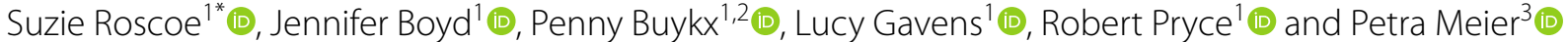

\begin{abstract}
Background: In the context of substantial financial disinvestment from alcohol and drug treatment services in England, our aim was to review the existing evidence of how such disinvestments have impacted service delivery, uptake, outcomes and broader health and social implications.

Methods: We conducted a systematic review of quantitative and qualitative evidence (PROSPERO CRD42020187295), searching bibliographic databases and grey literature. Given that an initial scoping search highlighted a scarcity of evidence specific to substance use treatment, evidence of disinvestment from publicly funded sexual health and smoking cessation services was also included. Data on disinvestment, political contexts and impacts were extracted, analysed, and synthesized thematically.

Results: We found 20 eligible papers varying in design and quality including 10 related to alcohol and drugs services, and 10 to broader public health services. The literature provides evidence of sustained disinvestment from alcohol and drug treatment in several countries and a concurrent decline in the quantity and quality of treatment provision, but there was a lack of methodologically rigorous studies investigating the impact of disinvestment.

Conclusions: This review identified a paucity of scientific evidence quantifying the impacts of disinvestment on alcohol and drug treatment service delivery and outcomes. As the global economy faces new challenges, a stronger evidence base would enable informed policy decisions that consider the likely public health impacts of continued disinvestment.
\end{abstract}

\section{Background}

Addressing the burden of alcohol and drug harm through the provision of treatment is a global priority [1]. Treatment for substance use disorders reduces health and social harms from alcohol and drugs, providing a good return on investment [2-9]. Many countries which publicly fund alcohol and drug services have been faced with large reductions in spending power, resulting in disinvestment from alcohol and drug treatment [10-13].

\footnotetext{
*Correspondence: smroscoe1@sheffield.ac.uk

1 School of Health and Related Research, University of Sheffield, Regent

Court, 30 Regent Street, Sheffield S1 4DA, UK

Full list of author information is available at the end of the article
}

In England, increased investment in treatment in the early twenty-first century, was associated with improved treatment access, reduced waiting times, improved service quality and a reduction in related harm [14-16]. Since 2012, there have been substantial changes to how drug and alcohol treatment in England is funded. The Health and Social Care Act 2012 transferred public health responsibilities, including the budget for alcohol and drug treatment, from the National Health Service to Local Authorities (local government organisations; N. 152 in England) [17]. At the same time a ring-fence protecting the alcohol and drug budget was removed, although protection for the total public health budget remained [18]. This transfer coincided with a period of 
public sector austerity in the wake of the global recession, with significant budget reductions for local government across a wide range of responsibilities [19, 20].

There have been widely reported changes to the investment in alcohol and drug treatment since 2014/15, with overall reductions in the amount local governments are investing in these services [21, 22]. Concurrently, trends in routine monitoring data show declines in treatment outcomes and increases in alcohol and drug related deaths and alcohol-related hospital admissions, with substantial variation across the country [23-25].

Whilst there is a strong evidence base for the effectiveness, and return on investment, of alcohol and drug treatment, the impact of recent disinvestment from these services remains unclear. Therefore, it is of policy interest and timely to synthesise available literature. An initial scoping search focused on alcohol and drug treatment revealed a paucity of evidence and therefore this review also considers what can be learnt from literature about disinvestments from similar local authority public health services, namely sexual health and smoking cessation services, which have also faced cuts [26, 27].

This review addressed the following questions:

i. What is the impact of disinvestment from publicly funded alcohol and drug treatment for adults in England?

ii. What is the impact of disinvestment from publicly funded alcohol and drug treatment for adults in other Organisation for Economic Co-operation and Development (OECD) countries?

iii. What can we learn from the impact of disinvestment from other publicly funded public health programmes, specifically smoking cessation and sexual health programmes, in England and other OECD countries?

\section{Methods}

\section{Protocol, registration and search strategy}

Following an initial scoping search, a pre-specified protocol was developed and registered on the International Prospective Register of Systematic Reviews (PROSPERO, CRD42020187295). We undertook a systematic search of the following bibliographic databases in July 2020: EMBASE (1980 to June 2020), MEDLINE (1946 to June 2020) and CINAHL (1981 to June 2020). An extensive list of search terms was used against each of the above research questions. To identify additional relevant, including grey, literature backward searching of citations was completed and www.evidence.nhs.uk and Google Scholar were searched using simplified search terms, for example, "cuts to alcohol and drug treatment".

\section{Inclusion criteria}

Journal publications and grey literature pertaining to the review questions and search strategy were included. This included primary and secondary quantitative and qualitative research examining the impact of disinvestment from the following publicly funded services: alcohol and drug, sexual health and stop smoking services. Relevant journal-published opinion pieces and grey literature from credible sources were also included. Any described or measured impacts related to disinvestment were included - for example, changes to the way services were commissioned or provided, treatment access and completion rates, and broader health and social implications. Sexual health and smoking cessation literature was included to enable learning to be drawn from comparable, large investment services that may have experienced budget cuts [28]. Additional inclusion criteria were literature that was: published in English; focused on OECD countries; services publicly funded for example, by a government body or a national health organisation.

\section{Data extraction and analysis}

Titles and abstracts of citations were screened within the bibliographic databases and those meeting the eligibility criteria were imported to EndNote, and duplicates were removed. Full texts were reviewed to dictate inclusion or exclusion before a data extraction table was compiled. Each paper was quality assessed using the most appropriate available tool for the reported study design via the Critical Appraisal Skills Programme (CASP) and the Joanna Briggs Institute (JBI) [29, 30]. The grey literature were appraised via the Authority Accuracy Coverage Objectivity Date Significance (AACODS) checklist [31]. The selection of the most appropriate critical appraisal tool was not always straightforward but is detailed within the supplementary information. For example, the Freudenberg et al. paper [32] was reviewed using the CASP systematic review checklist as the paper is a peerreviewed synthesis of relevant literature. However, it does not follow a systematic review design and therefore it is unclear whether all relevant papers were included, or if included papers were assessed for quality. Furthermore, the diversity of included publication types means that some were unlikely to have been written with quality appraisal in mind. For example, within the grey literature, the limitations and bias of the content covered (or the research undertaken) were not always explicit, which impacted on the ability to assess the overall accuracy of the papers.

The papers were then analysed thematically, adopting Braun and Clarke's approach to qualitative data [33], and synthesised narratively, using the Synthesis 
Without Meta-analysis protocol [34]. SR led the search, data extraction and analysis and JB reviewed all papers to confirm eligibility, and completed thematic analysis of half of the papers, prior to discussion and agreement of final themes. JB also independently quality appraised a random sample of $25 \%$ of included papers. Given the heterogeneity of the papers and that no study attempted to quantify the primary question, no weighting of results was applied according to, for example, whether claims are substantiated by empirical findings. Instead, an inductive thematic approach was used to explore conceptual similarities across heterogeneous literature to provide an overview of the politico-economic context of any disinvestments, related changes to provision and outcomes. The extraction tables (Tables 1 and 2) provide details of the publication and / or study type.

\section{Results}

\section{PRISMA diagram}

Figure 1 shows the flow of articles through the review process. Database and grey literature searches returned 1812 records; of which 196 underwent full text screening. Twenty papers were included in the review.

\section{Settings and quality of papers \\ Study characteristics and quality}

Of the 20 eligible papers, 13 were research papers, five journal editorials and two substance misuse professional magazine articles. Ten papers related to alcohol and drugs services, three to sexual health services, two to smoking cessation services and five to public health services more generally. Table 1 provides data extracted from the papers explicitly focused on disinvestment from alcohol and drug treatment services and Table 2 shows data from the wider papers. Four of the research papers were peer reviewed: one English study analysing results from a survey of local government tobacco leads regarding smoking cessation services [35], two US studies exploring data and literature on specific public health policy and funding [32, 36], and one Japanese study analysing secondary survey and routine finance data examining the relationship between (dis)investment and smoking cessation advice [37]. Six of the remaining research papers focused on substance use $[15,38-41]$ and were UK $(n=5)$ and multi-country European $(n=1)$ based. One of the five journal editorials [42] and both magazine articles [43, 44] were substance use specific, the remainder focussed on broader public health services. The majority of these were from the UK (UK $n=6$, Australia $n=1$ ). The overall quality of included papers according to quality appraisal was modest. However, due to the limited number of relevant papers identified, no papers were excluded on the basis of low quality. No studies that attempted to examine a quantifiable or causal relationship between disinvestment from substance use services and treatment delivery or outcomes were identified. Instead, the studies tend to focus on changes in treatment provision and related health outcomes, concurrent or subsequent to disinvestment.

\section{Thematic synthesis}

Three major themes were identified: i) diminished quantity and quality of services; ii) changed commissioning systems and practices; and iii) health, social and broader implications. We present findings relating to each of these themes in turn.

\section{Diminished quantity and quality of services}

The literature offers insights to how services offered have changed in the wake of disinvestment, often relating a decline in the availability of treatment and a deterioration in the quality of support offered $[10,15,32,38,40,41,43$, 45-47].

Initial cuts to alcohol and drug treatment services were purported to have provided opportunities to find efficiencies and drive service reform [15], and to focus on a greater return on investment [47]. However, continued cuts were described as detrimental to service availability and quality $[15,38,40,41]$. Organisational research details stakeholder concern that the funding available for alcohol and drug treatment has become increasingly insufficient $[15,39,47]$, and is mismatched to the vision for "gold-standard" treatment services in recent clinical guidelines $[15,21,43]$.

As budget cuts continued, specific interventions and treatment modalities including harm reduction [41, 44] and residential rehabilitation [39] were regarded as under particular threat. Mixed methods studies targeting treatment sector stakeholders revealed concerns about increasing caseloads, fewer appointments, the replacement of one-to-one work with group sessions, reduced harm reduction and less outreach support $[15,38,39,41$, 45-47]. Similar changes have been experienced in smoking cessation and sexual health services following disinvestment, referencing a propensity to focus on acute care when budgets are tight [49-51]. This latter concern has also been raised specifically in relation to the alcohol and drug sector, suggesting that services were having to revert to focussing solely on maintenance prescribing [43].

In addition to changes in the treatment offered, there were reports of a reduction in the number of people accessing $[15,32,40]$ and successfully completing alcohol and drug treatment [47]. This echoes experiences following disinvestment from sexual health services in the UK [50,51], from drug treatment in the US [32], and from smoking cessation support in Japan [37]. In Japan, 


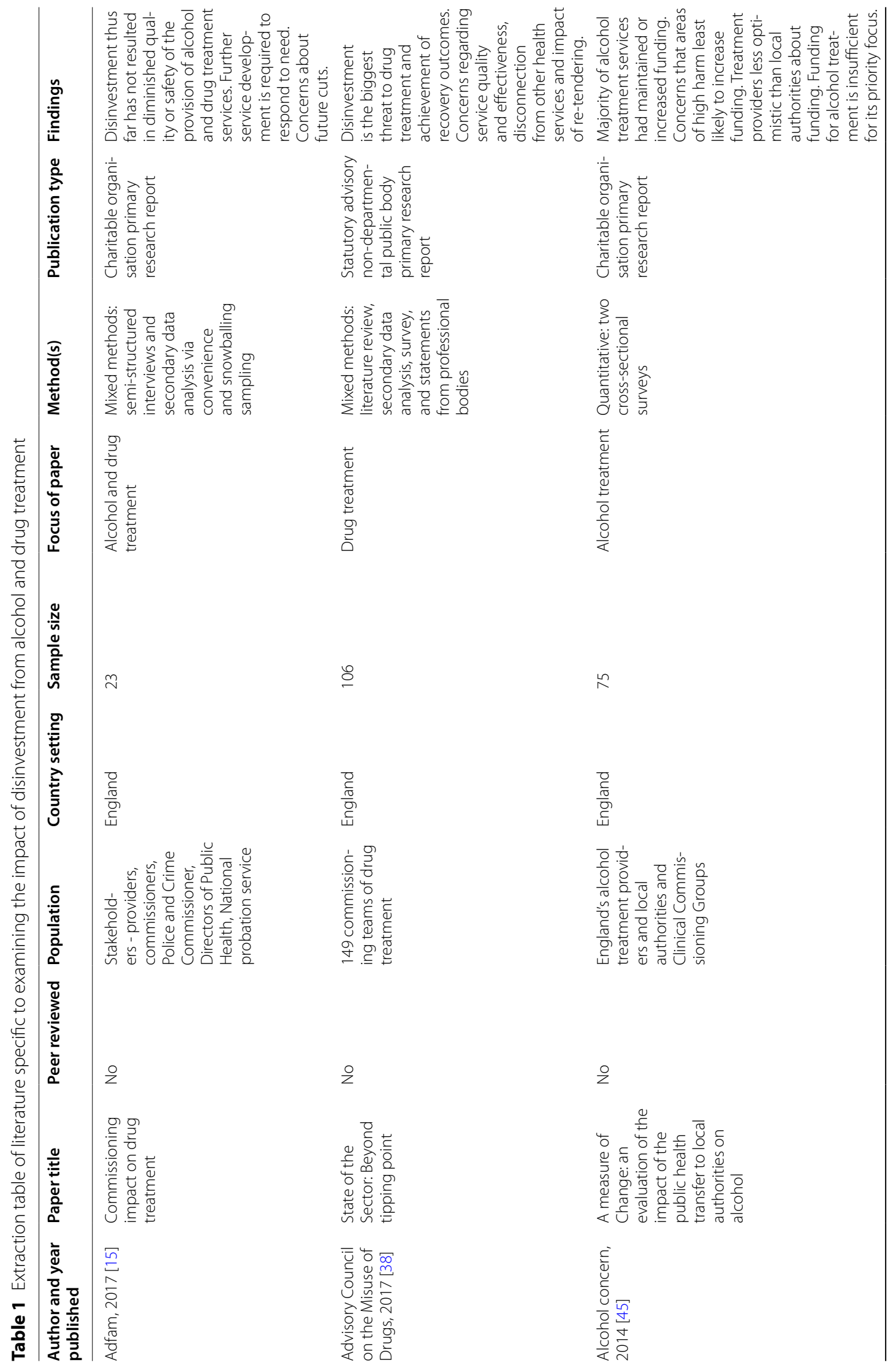




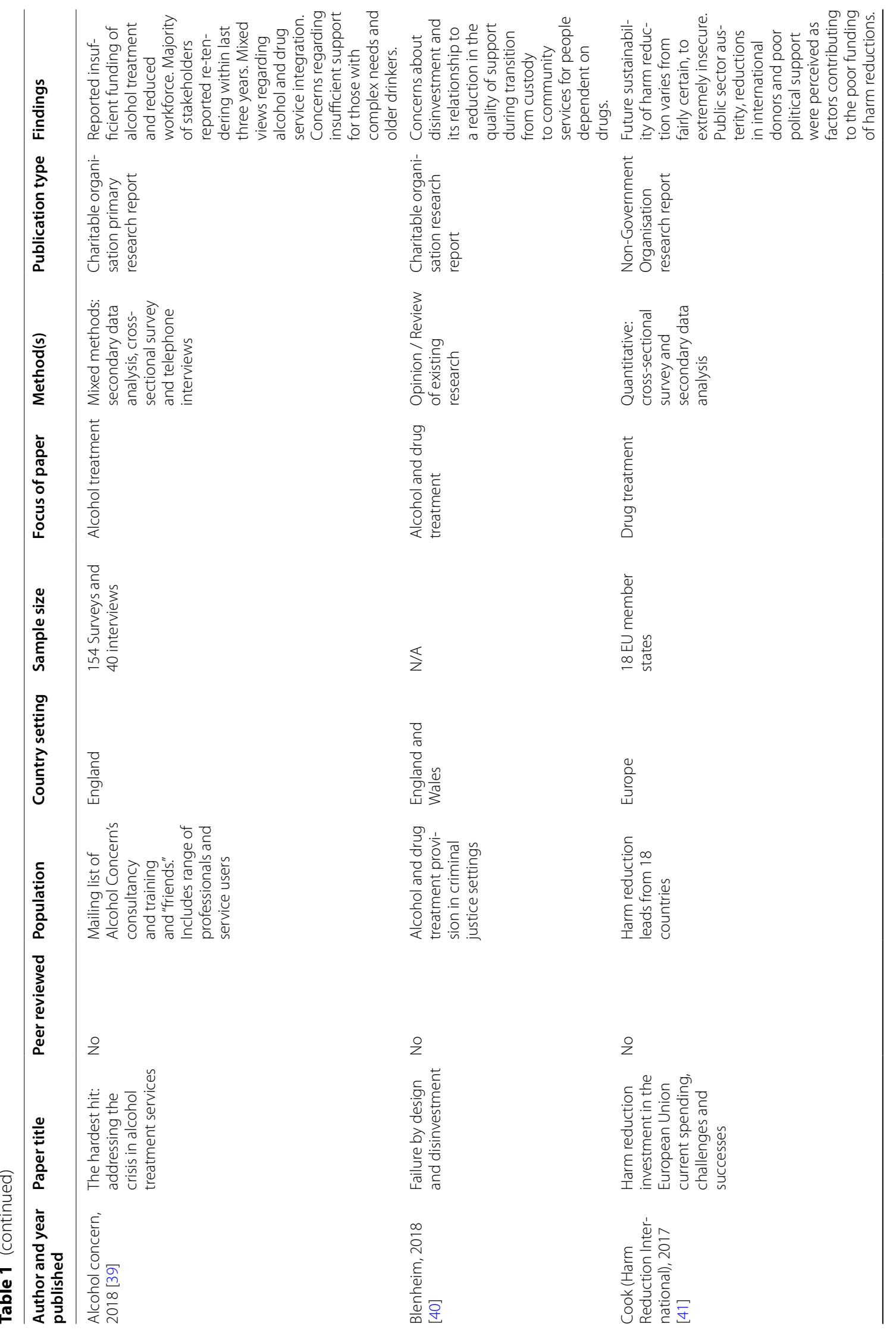




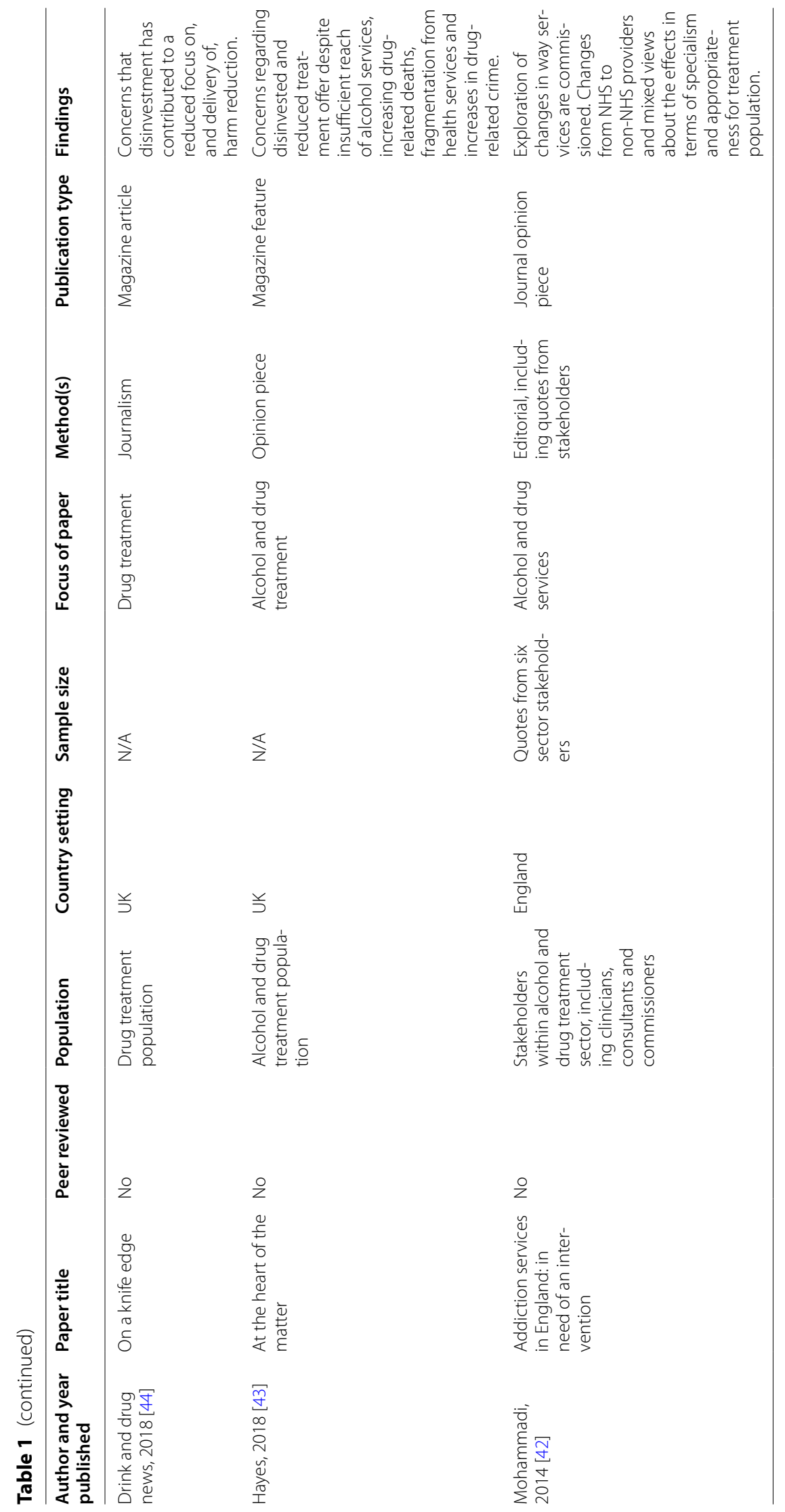




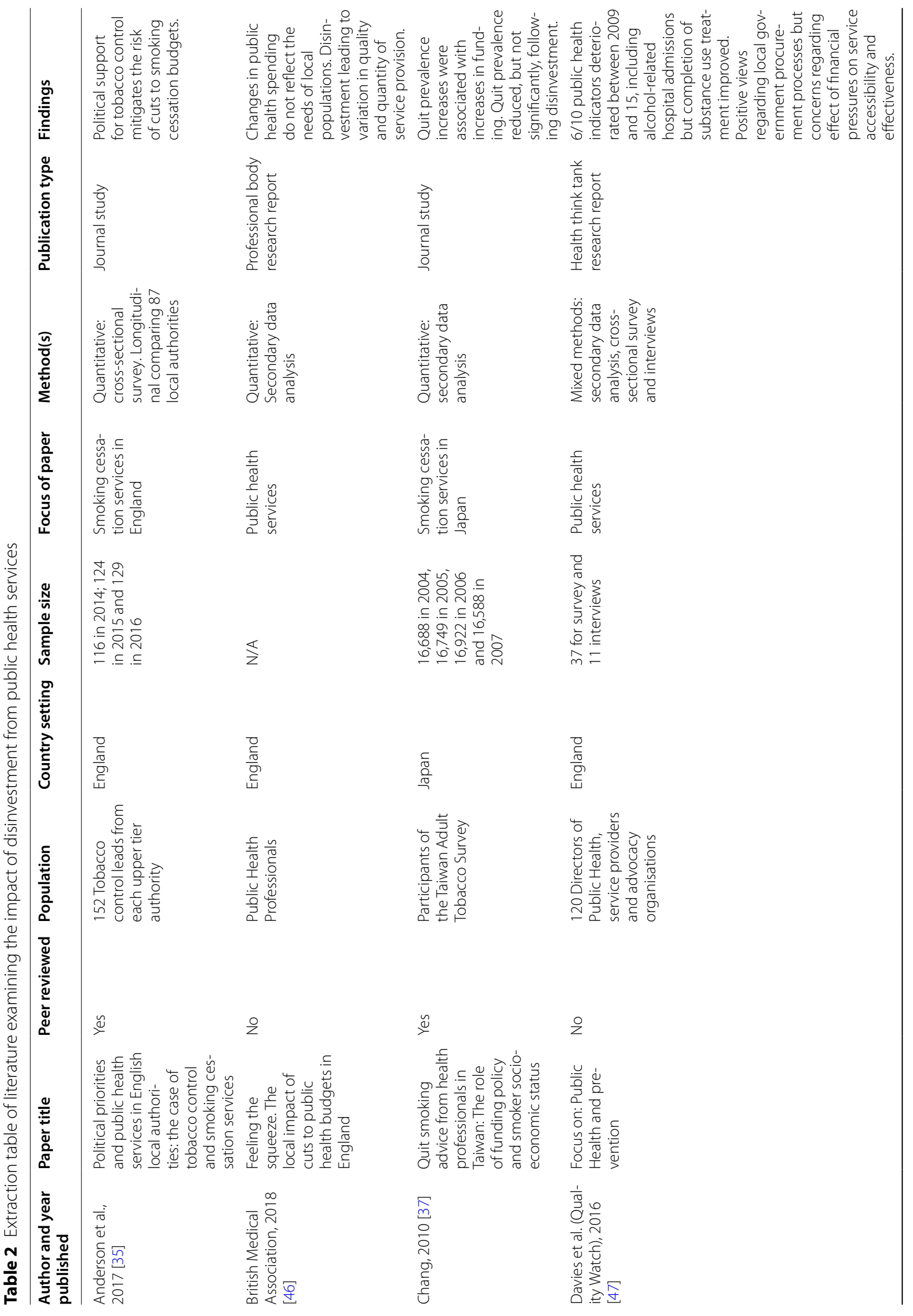




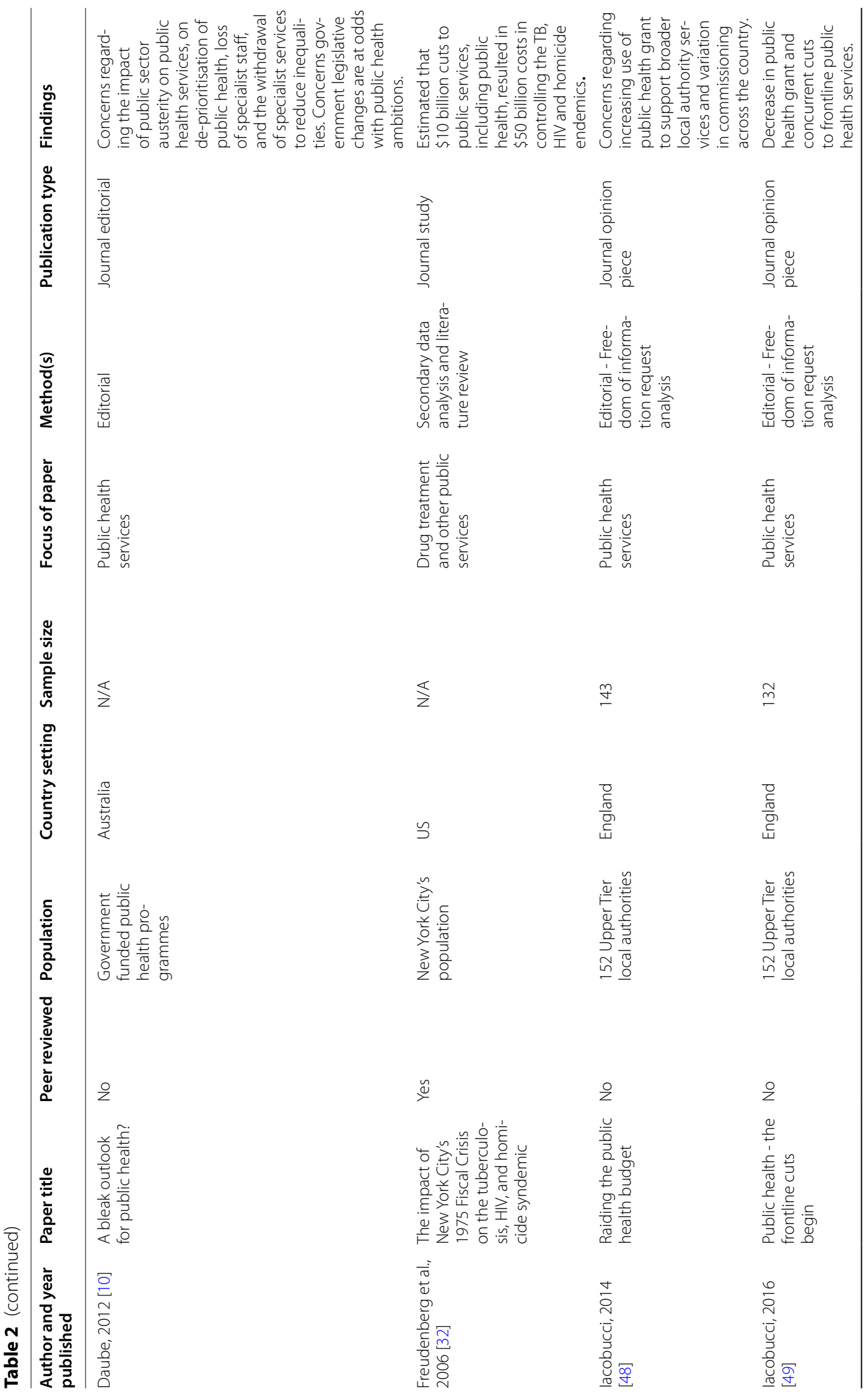




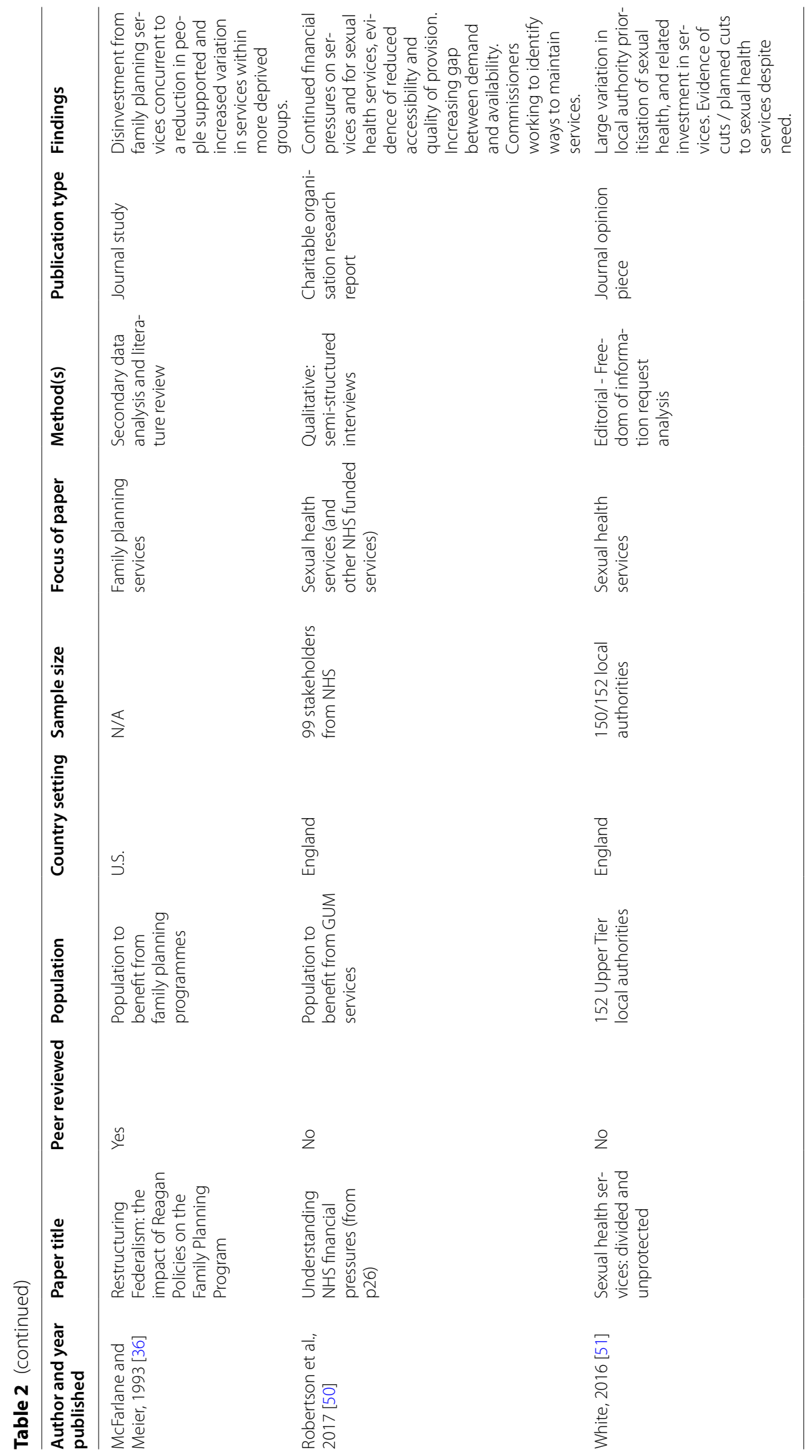




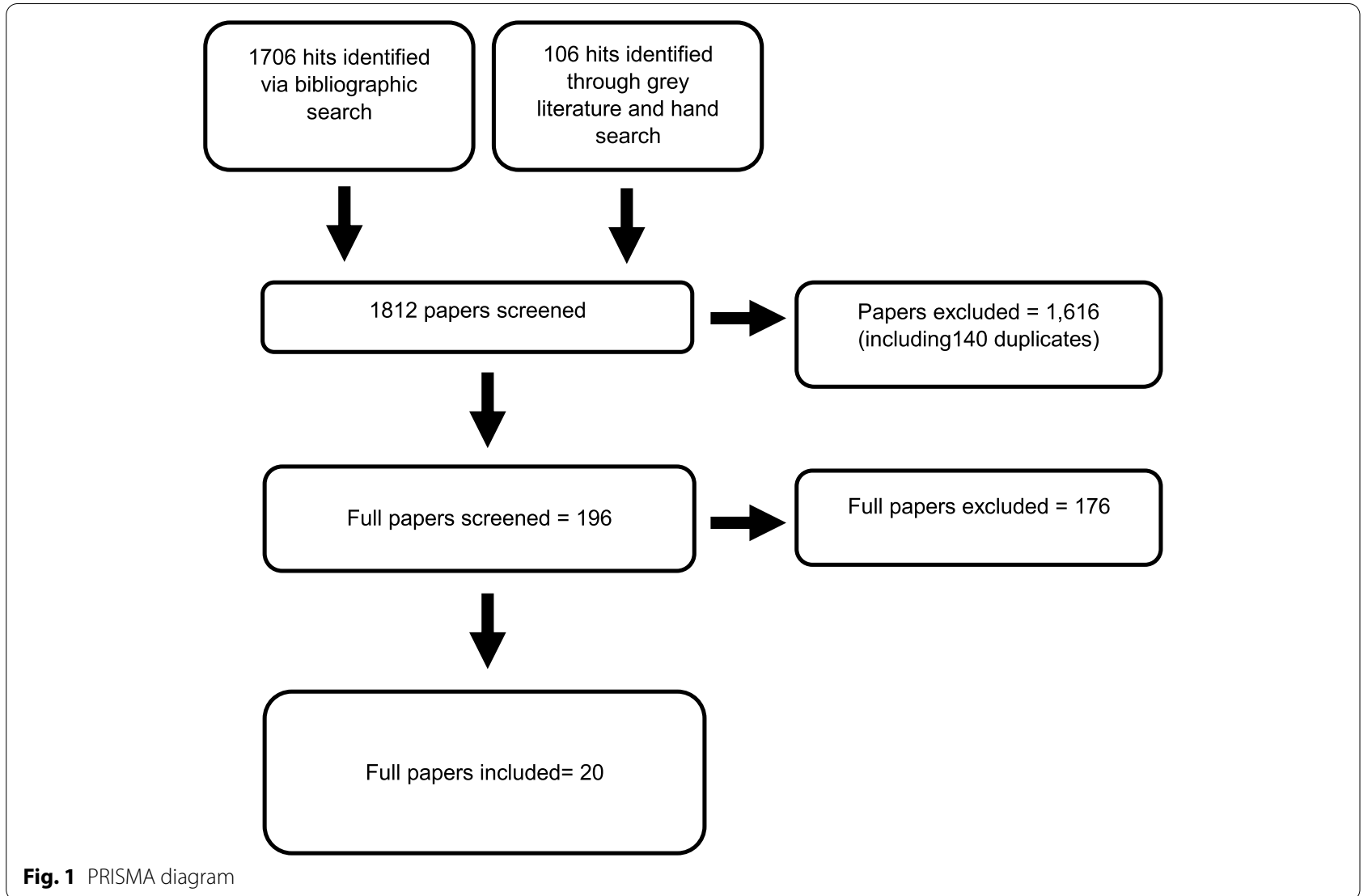

additional effects were seen following disinvestment, including reduced stakeholder engagement and fewer smoking cessation media campaigns [37].

Substantial changes in the alcohol and drug treatment sector during a period of disinvestment were purported to have contributed to an increasingly deskilled and disenfranchised workforce [15, 32, 38]. This included examples of an overreliance on volunteers who had replaced paid staff [15, 38], a loss of specialist positions (such as addiction psychiatrists for more generic clinician roles) [42], and a reduction in the amount of training for the sector's workforce $[15,37,38,42]$.

\section{Changed commissioning systems and practices}

The processes and systems that exist to commission public health services also appeared to have changed substantially. Subsequent to the transfer of public health responsibilities to local authorities, the stretch on financial resources affected commissioning systems and practices [15, 32, 35, 39, 41, 46]. This included resulting changed responsibilities, procurement activity and fragmentation, with large variation across local authorities.

A growing number of local government areas in England are reported to have integrated various public health services into combined contracts, including the merger of community alcohol and community drug services $[15,39,48]$. Limited attention has been given to the rationale for this move but budget efficiencies are cited in some cases [36, 40], and these mergers have been criticised for reducing service effectiveness [36, 49]. Alcohol and drug treatment sector stakeholders raised concerns that integration can weaken evidencebased practice and that the merger of alcohol and drug services might result in a disproportionate, or diluted, provision for the alcohol treatment population [39].

Whilst it is unclear as to whether the number of retendering exercises has increased, the frequency and process of retendering of alcohol and drug services has been described as hindering outcomes and detracting from frontline delivery of services for a period of up to 18 months [15, 38-40,42, 47, 48]. There has also been a rise in the use of payment by results, aligning all or partial contract payment to the achievement of specific goals, such as abstinence. Though recognised as an option for achieving a greater return on investment, such payment schedules are perceived as side-lining a client group for whom abstinence is not a goal $[38,42]$. 
Disinvestment has been linked to a reduction in the number of service providers able to bid for treatment contracts $[15,38,50]$. The reduced budgets available to finance contracts is perceived as favouring non-National Health Service (NHS) to NHS providers [42]. It is also been linked to a reduction in the number of organisations applying for treatment contracts, excluding smaller local organisations and the evolution of treatment systems led by national organisations [15].

Meanwhile, the expertise of alcohol and drug treatment commissioners in England is under scrutiny [15, 39] with feedback from stakeholders that subject-specific expertise has been lost from commissioning teams as a result of staff turnover and an increase in the size and scope of commissioners' portfolios [10, 15]. This is echoed in sexual health services which have been criticised as fragmented, with disjointed services and an increasing lack of accountability [50]. This includes examples of different aspects of services being commissioned via different bodies with diverse procurement approaches, resulting in disjointed pathways. This fragmentation in commissioning arrangements has also been criticised as leading to isolated disinvestment decisions, especially when cuts to one service have knock-on implications for other parts of the system.

A further contention within the local authority environment for public health is the fit with local political agendas [32, 36, 37, 42, 45, 47-49, 51]. Decisions about investment in a context of competing policy areas [49], investment choices being driven by popularity [38, 51], and not being able to align the benefits of public health services with local authority strategy [48] all appear to factor. Such differences across local authorities have been described as contributing to large variations in the prioritisation of public health agendas, investment and service provision $[36,48,51]$.

\section{Health, social and other broader negative implications}

Disinvestment from public health services has led to concerns about a downstream rise in demand on other publicly-funded services, and increases in communicable disease and crime [15, 32, 36, 39-41, 43, 45-47]. Editorials have highlighted that concurrent to disinvestment from other public health services, there have been deteriorating related outcomes, including increased rates of sexually transmitted diseases and teenage pregnancies, and a stagnation of the narrowing of socioeconomic gaps in life expectancy and quality of life $[42,48,49,51]$.

One English study, analysing routinely-collected secondary data, expressed concern about such disproportionate cuts to public health services contributing to widening health inequalities, with large variation in the quantity and quality of services available [46]. In a historical health impact study in the US [36], poorer health outcomes for low-income women were attributed to $30 \%$ cuts to family planning services .

Simultaneous to disinvestment from the alcohol and drug treatment sector have been increases in alcohol related hospital admissions and drug related deaths [15, 43, 45-47]. A historical health impact study in the US identified that policy decisions and budget cuts to public health services led to reduced availability of drug treatment [32]. The exponential rise in tuberculosis and HIV within the injecting drug treatment population - although the relationship was not formally analysed or modelled - was attributed to these budget cuts. Similar concerns have been raised in England more recently concerning the increasing number of drug-related deaths relating to fentanyl and how they might be linked to reduced needle exchange provision and associated support [44].

Furthermore, disinvestment appears linked to the withdrawal, or dilution, of services that support vulnerable groups [10, 40, 43]. For example, large disinvestment from substance use prison services has been linked to a lack of supported transition to community treatment, poor case management and a lack of Naloxone, potentially contributing to the rise in drug-related deaths [10, 40]. Similarly, people who may have previously benefited from targeted programmes [50] appear further marginalised following policy changes, including people in ethnic minority groups [10], people experiencing mental ill health and those with housing needs $[10,43]$.

\section{Discussion}

The understanding of the impact of disinvestment is limited and no previous study has systematically examined the evidence. This study synthesises heterogeneous papers that provide insight as to how disinvestment from public health services might affect service provision and outcomes. Twenty papers were identified that contribute to understanding the impact of disinvestment from alcohol and drug treatment, and related public health services, in England and elsewhere. The review identified similarities between the described effects of disinvestment from alcohol and drug treatment services with the effects of disinvestment from broader public health services. The broader papers provide some additional empirical evidence in support of this review's identified themes, including for example, poorer outcomes [37] and the effects of political influence [35].

Policy makers are facing challenging public health investment decisions during a time of sustained public austerity. There are numerous reported changes to the way services have been commissioned which may have negatively influenced treatment quality. Whilst perhaps driven by a need for efficiencies, service integration 
may have limited the specialisms within workforces and disproportionately impacted the alcohol treatment population.

The literature highlights concerns about the reduced quantity and quality of alcohol and drug treatment in England, following cuts to services. This is echoed in literature from other OECD countries and literature on disinvestment from other, similar public health services. However, there is limited exploration as to whether certain changes, including for example the integration of alcohol and drug treatment services, were done to limit direct impact of budget reductions. This study also identifies some evidence that disinvestment might be impacting more on some of the most disadvantaged areas, and vulnerable communities, potentially contributing to increasing health inequalities. Certain aspects of the treatment system are reported to have been disproportionately affected by budget cuts. Fewer harm reduction services and residential rehabilitation facilities, and less one on one time, may present particular challenges for people with more complex needs [21].

The influence of political agendas and competing pressures - where investment decisions are devolved - may be contributing to inconsistent investment and treatment provision. Disinvestment was often described in relation to the context of public sector austerity $[15,35,36$, $43,46,47]$ and how some cuts have been disproportionate to need [10, 38, 46, 52]. An English study highlighted an $8 \%$ reduction in expenditure on substance use services versus a $5 \%$ reduction in the available public health grant between 2013/14 and 2017/18 [38]. Two studies and an opinion piece also highlighted that local changes in investment in public health services in England had varied substantially between local authorities [39, 46, 51]. Some of the areas that had experienced the highest levels of alcohol and drug-related harm had reported some of the biggest percentage cuts to service budgets [43, 45, 46]. Investment decisions have been reported as being guided by political priorities and even personal stigmatisation of treatment populations [10, 32, 35, 37, 38, 41]. Given these concerns, and evidence that some vulnerable people may be being disproportionately affected by changes to treatment provision, it may be that disinvestment is contributing to widening health inequalities [53, 54].

Further to the themes identified in this review regarding the impact of disinvestment, there were substantial references within the literature to the context and conditions of disinvestment. Previous increases in investment were reported to have enabled innovation, for example, increased psychosocial support for people with alcohol and drug dependence and embedded support services within community settings [50]. Despite a reported substantial rise in investment in alcohol treatment between 2013/14 and 2015/16 [15], some claims were made within the literature that funding for alcohol has always been insufficient, with over two thirds of amalgamated budget being spent on drug treatment $[15,39,43$, 45].

Furthermore, the funding mechanisms devised to help protect public health grant funding in England (such as ring-fencing, to prevent expenditure on non-public health services) appear to have been limited in their success $[15,35,38,47,48,50]$. These UK papers report public health grant funding being utilised to subsidise other local authority service provision, such as domestic abuse services, that do not fall within current statutory public health responsibilities. Within a context of local authority austerity, six papers highlighted stakeholder concerns that pressures on public health spending in the UK would further increase [15, 38, 41, 46, 47, 51], due to an expected decreasing public health grant and the intended removal of the ring-fence.

\section{Limitations of the study}

The heterogeneity of the papers, in terms of the research methods employed and the way in which information was analysed and presented, limited our ability to synthesise results or make comparisons, leading us to choose a narrative-interpretive approach. The focus of this review and synthesis of diverse literature means that some of the results from individual papers will not have been detailed. The alcohol and drug treatment papers often failed to clearly outline the objectives or proposed analyses of their studies and therefore lacked transparency as to the measured outcomes or the criteria used to assess impact. This made it difficult to differentiate impacts associated with disinvestment from impacts associated with simultaneous commissioning, service provision and policy changes, or indeed the drivers of those changes. Whilst the literature about England clearly reports financial disinvestment from alcohol and drug treatment services and the perceived impact of these cuts, the association between the two and the accuracy of the published financial information, have not been studied. Furthermore, the drivers of disinvestment remain unclear, and how cuts have impacted on different elements of the treatment system, for example, different treatment modalities, or the configuration of services.

\section{Future research}

This review has identified concepts which further empirical research should seek to examine to further advance the evidence of the impact of disinvestment from alcohol and drug treatment services, and other public health services. In England, for example, there are substantial 
routine data available to quantitatively examine the effects of disinvestment on treatment access and outcomes, as well as additional broader health harms. In countries where such data is available, it could be matched on a local geography or where available, matching patient and treatment data. This could help us to better understand variation in disinvestment and relative changes in treatment availability and effectiveness. As the systems that enable treatment appear complex and vary substantially, qualitative methods with key stakeholders could identify additional factors contributing to the effect of disinvestment. Within the reviewed literature, there is limited reference to attempts to moderate the impact of disinvestment and yet there are references to innovation in commissioning practices and service delivery during a period of sustained cuts. Further exploration of these factors may be helpful to support future decision-making to maintain treatment engagement and quality.

The important contextual factors to (dis)investment, regularly referenced within the literature, could be considered in future studies. For example, examining regional or socioeconomic variation in (dis)investment and treatment provision would help further advance our understanding as to whether budget cuts may be disproportionately affecting people living in deprived areas. Furthermore, research which seeks to understand local drivers of (dis)investment in alcohol and drug treatment services may also help to identify protective factors.

The quality appraisal of included research studies the literature highlighted some weaknesses in terms of study design and transparency in reporting. Therefore, future research should seek to fully report methods and use a quality checklist to improve its robustness.

\section{Conclusions}

This study is the first to synthesise literature that explores the impact of disinvestment on alcohol and drug treatment and outcomes and identifies opportunities to further advance the body of evidence. In England, disinvestment from alcohol and drug treatment services has occurred in parallel to reduced public sector funding, declines in treatment outcomes and increases in alcoholrelated hospital admissions and alcohol and drug-related deaths. However, the quantitative relationship between disinvestment from alcohol and drug treatment and related outcomes remains unexamined. Since the Health and Social Care Act 2012, substantial changes to the way in which services are commissioned and provided were reported. There was evidence of large variation in disinvestment across England with concerns about the potential for widening health inequalities. Given the known link between effective alcohol and drug treatment and reduced health and social harms, understanding the impact of disinvestment remains important to policy makers internationally. This may be particularly important given that disinvestment might result in increased pressure on more costly publicly funded services.

\section{Supplementary Information}

The online version contains supplementary material available at https://doi. org/10.1186/s12889-021-12219-0.

Additional file 1.

\section{Acknowledgements}

Not applicable.

\section{Authors' contributions}

SR led conceptualisation and development of the review, with input from LG and PB. SR led the search, review and quality appraisal. JB reviewed all papers to confirm eligibility, and completed thematic analysis of half of the papers, prior to discussion and agreement of final themes. JB also independently quality appraised a random sample of $25 \%$ of included papers. SR was a major contributor in writing the manuscript and SR, JB, PB, LG, RP and PM read, edited and approved the final manuscript.

\section{Funding}

This work was supported by funding from The Wellcome Trust (Award number 108903/B/15/Z). For the purpose of Open Access, the author has applied a CC BY public copyright license to any Author Accepted Manuscript version arising from this submission.

Availability of data and materials

Not applicable.

\section{Declarations}

Ethics approval and consent to participate

Not applicable.

Consent for publication

Not applicable.

Competing interests

The authors declare that there are no competing interests.

\section{Author details}

${ }^{1}$ School of Health and Related Research, University of Sheffield, Regent Court, 30 Regent Street, Sheffield S1 4DA, UK. ${ }^{2}$ School of Humanities and Social Science, University of Newcastle, University Drive, Callaghan, NSW 2308, Australia. ${ }^{3} \mathrm{MRC} / \mathrm{CSO}$ Social and Public Health Sciences Unit, University of Glasgow, Berkeley Square, 99 Berkeley Street, Glasgow G3 7HR, UK.

Received: 29 April 2021 Accepted: 14 October 2021

Published online: 22 November 2021

\section{References}

1. World Health Organization. Stronger collaboration, better health: global action plan for healthy lives and well-being for all. 2019. Available from: https://www.who.int/publications-detail/stronger-collaboration-betterhealth-global-action-plan-for-healthy-lives-and-well-being-for-all.

2. Ferri M, Davoli M, Perucci CA. Heroin maintenance treatment for chronic heroin-dependent individuals: a Cochrane systematic review of effectiveness. J Subst Abus Treat. 2006;30(1):63-72.

3. Gowing L, Farrell M, Bornemann R, Sullivan L, Ali R. Substitution treatment of injecting opioid users for prevention of HIV infection. Cochrane 
Database Syst Rev. 2008;Issue 2. Art. No.: CD004145. https://doi.org/10. 1002/14651858.CD004145.pub3. Accessed 19 Nov 2021.

4. MacArthur GJ, Minozzi S, Martin N, Vickerman P, Deren S, Deghenhardt $L$, et al. Opiate substitution treatment and HIV transmission in people who inject drugs: systematic review and meta-analysis. Br Med J. 2012;345:e5945.

5. Martin GW, Rehm J. The effectiveness of psychosocial modalities in the treatment of alcohol problems in adults: a review of the evidence. Can J Psychiatr. 2012:57(6):350-8.

6. Mattick RP, Breen C, Kimber J, Davoli M. Methadone maintenance therapy versus no opioid replacement therapy for opioid dependence. Cochrane Database Syst Rev. 2009;(3) Available from: http://doi.wiley.com/10.1002/ 14651858.CD002209.pub2. [cited 2020 Sep 11].

7. Ryder SD, Aithal GP, Holmes M, Burrows M, Wright NR. Effectiveness of a nurse-led alcohol liaison service in a secondary care medical unit. Clin Med (Northfield II). 2010;10(5):435-40.

8. Turner KME, Hutchinson S, Vickerman P, Hope V, Craine N, Palmateer N, et al. The impact of needle and syringe provision and opiate substitution therapy on the incidence of hepatitis C virus in injecting drug users: pooling of UK evidence. Addiction. 2011;106:1978-88.

9. NICE. Alcohol-use disorders: diagnosis, assessment and management of harmful drinking and alcohol dependence. Clinical guideline CG115: NICE; 2011. Available from: https://www.nice.org.uk/guidance/cg115. Accessed 3 Aug 2020.

10. Daube M. A bleak outlook for public health? Aust N Z J Public Health. 2012;36(6):503-4.

11. European Monitoring Centre for Drugs and Drugs Addiction. Drug treatment expenditure. 2017. Available from: http://www.emcdda.europa.eu/ publications/insights/drug-treatment-expenditure-measurement_en.

12. Ministry of Housing Communities and Local Government. Financial sustainability of local authorities 2018. 2018. Available from: https://www. nao.org.uk/report/financial-sustainability-of-local-authorities-2018/.

13. Local Government Association. Local government funding: moving the conversation on. 2018. Available from: www.local.gov.uk/moving-theconversation-on.

14. Collective Voice. Briefing for health select committee roundtable with practitioners. 2016. Available from: https://www.collectivevoice.org.uk/ blog/briefing-health-select-committee/. [cited 2020 Aug 12].

15. Adfam. State of the sector 2017: beyond the tipping point. 2017. Available from: http://www.recovery-partnership.org/uploads/5/1/8/2/51822 429/state_of_the_sector_2017_-_beyond_the_tipping_point.pdf.

16. Public Health England. An evidence review of the outcomes that can be expected of drug misuse treatment in England. 2017. Available from: https://assets.publishing.service.gov.uk/government/uploads/system/ uploads/attachment_data/file/586111/PHE_Evidence_review_of_drug_ treatment_outcomes.pdf. [cited 2021 Feb 6].

17. Health and Social Care Act 2012, c. 7. Available at: http://www.legislation. gov.uk/ukpga/2012/7/contents/enacted. Accessed 3 Aug 2020

18. Powell T, Baker C, Donnell MO. Opposition day debate : health and local public health cuts. 2019. Available from: https://researchbriefings.files. parliament.uk/documents/CDP-2019-0121/CDP-2019-0121.pdf.

19. Local Government Association. Health and local public health cuts, house of commons, 14 may 2019; 2019. p. 1-4.

20. Institute for Government. Local government funding in England. 2020. Available from: https://www.instituteforgovernment.org.uk/explainers/ local-government-funding-england.

21. Independent Expert Working Group. Drug misuse and dependence: UK guidelines on clinical management. 2017. Available from: https://assets. publishing.service.gov.uk/government/uploads/system/uploads/attac hment_data/file/673978/clinical_guidelines_2017.pdf.

22. HM Government. 2017 drug strategy. 2017. Available from: https://assets. publishing.service.gov.uk/government/uploads/system/uploads/attac hment_data/file/628148/Drug_strategy_2017.PDF.

23. Office for National Statistics. Deaths related to drug poisoning in England and Wales: 2015 registrations. Health statistics quarterly. 2016. Available from: https://www.ons.gov.uk/peoplepopulationandcommunity/birth sdeathsandmarriages/deaths/bulletins/deathsrelatedtodrugpoisoningin englandandwales/previousReleases. [cited 2020 Aug 11].

24. Public Health England. Adult substance misuse treatment statistics 2018 to 2019 report. 2019. Available from: https://www.gov.uk/gover nment/publications/substance-misuse-treatment-for-adults-stati stics-2018-to-2019/adult-substance-misuse-treatment-stati stics-2018-to-2019-report.

25. Public Health England. Public health profiles. 2018. Available from: https://fingertips.phe.org.uk/search/alcoholmortality\#page/3/gid/1/ pat/6/par/E12000005/ati/102/are/E06000021/iid/91382/age/1/sex/4. [cited 2018 May 14].

26. Advisory Group on Contraception. Cuts, closures and contraception - an audit of local contraceptive services in England. 2017. Available from: http://theagc.org.uk/wp-content/uploads/2018/03/AGC_Report-Final2017.pdf.

27. Cancer Research UK, Action on Smoking and Health. A changing landscape for stop smoking services and tobacco control in England. 2019. Available from: http://ash.org.uk/wp-content/uploads/2019/03/2019-LASurvey-Report.pdf.

28. Ministry of Housing Communities and Local Government. Local authority revenue expenditure and financing England: 2015 to 2016 final outturn. 2016. Available from: https://www.gov.uk/government/statistics/localauthority-revenue-expenditure-and-financing-england-2015-to-2016final-outturn. [cited 2018 Feb 23].

29. Joanna Briggs Institute. Critical appraisal tools. 2018.

30. Critical Appraisal Skills Programme. CASP Checklists. 2017.

31. Tyndall J. The AACODS checklist is designed to enable evaluation and critical appraisal of Grey literature. Chin J Evid Based Med. 2010;7(1):50713 Available from: https://dspace.flinders.edu.au/xmlui/bitstream/ handle/2328/3326/AACODS_Checklist.pdf;jsessionid=F726F414C378EBE B2DB31F17F88A75FE? sequence $=4$.

32. Freudenberg N, Fahs M, Galea S, Greenberg A. The impact of New York City's 1975 fiscal crisis on the tuberculosis, HIV, and homicide syndemic. Am J Public Health. 2006;96(3):424-34.

33. Braun V, Clarke V. Using thematic analysis in psychology. Qual Res Psychol. 2006:3(2):77-101.

34. Campbell M, McKenzie JE, Sowden A, Katikireddi SV, Brennan SE, Ellis $\mathrm{S}$, et al. Synthesis without meta-analysis (SWiM) in systematic reviews: reporting guideline. BMJ. 2020;368:1-6.

35. Anderson WJ, Cheeseman H, Butterworth G. Political priorities and public health services in English local authorities: the case of tobacco control and smoking cessation services. J Public Health (Bangkok). 2017:40(3):1-6.

36. McFarlane DR, Meier KJ. Restructuring federalism: the impact of Reagan policies on the family planning program. J Health Polit Policy Law. 1993;18(4):821-50.

37. Chang FC, Hu TW, Lo SY, Yu PT, Chao KY, Hsiao ML. Quit smoking advice from health professionals in Taiwan: the role of funding policy and smoker socioeconomic status. Tob Control. 2010;19(1):44-9.

38. Advisory Council on the Misuse of Drugs. Commissioning impact on drug treatment. 2017. Available from: https://www.gov.uk/government/ publications/commissioning-impact-on-drug-treatment.

39. Alcohol Concern. The hardest hit. 2018. Available from: https://alcoholcha nge.org.uk/publication/the-hardest-hit-addressing-the-crisis-in-alcoholtreatment.

40. Blenheim CDP. Failure by design and disinvestment : the critical state of transitions; 2018.

41. Cook C. Harm reduction investment in the European Union: current spending, challenges and successes [internet]. 2017. Available from: https://www.hri.global/contents/1782.

42. Mohammadi D. Addiction services in England: in need of an intervention, 2014. www.thelancet.com/psychiatry.

43. Hayes P. The heart of the matter - drink and drugs news: Drink and Drug News; 2018. Available from: https://www.drinkanddrugsnews.com/ the-heart-of-the-matter-2/

44. Drink and Drug News. Harm reduction on a knife edge: Drink and Drug News; 2018. Available from: https://www.drinkanddrugsnews.com/on-aknife-edge/. [cited 2020 Aug 27]

45. Alcohol Concern. A measure of change. 2014

46. British Medical Association. Feeling the squeeze - the local impact of cuts to public health budgets in England; 2018. p. 1-14.

47. Davies A, Keeble E, Bhatia T, Fisher E. Focus on: public health and prevention has the quality of services changed over recent years? 2016.

48. lacobucci G. Raiding the public health budget. BMJ. 2014;348:-g2274.

49. Iacobucci G. Public health-the frontline cuts begin. BMJ. 2016;532:1272. 
50. Robertson R, Wenzel L, Thompson J, Charles A. Understanding NHS financial pressures: how are they affecting patient care? The King's Fund 2017. Available from: https://www.kingsfund.org.uk/sites/default/files/ field/field_publication_file/UnderstandingNHSfinancialpressures-fullr eport.pdf\%0A, https://www.kingsfund.org.uk/sites/files/kf/field/field_ publication_file/UnderstandingNHSfinancialpressures-fullr.

51. White C. Sexual health services: divided and unprotected. BMJ. 2016;352:1309-9.

52. DrugScope. State of the sector 2014-15. Summary report. 2015. Available from: https://www.basw.co.uk/resources/state-sector-2014-_-15.
53. Marmot $M$, Bell R. Fair society, healthy lives (full report). Public Health. 2012;126(1):S4-10 Available from: https://doi.org/10.1016/j.puhe.2012.05. 014.

54. Woodward A, Kawachi I. Why reduce health inequalities? J Epidemiol Community Health. 2000;54:923-9.

\section{Publisher's Note}

Springer Nature remains neutral with regard to jurisdictional claims in published maps and institutional affiliations.
Ready to submit your research? Choose BMC and benefit from:

- fast, convenient online submission

- thorough peer review by experienced researchers in your field

- rapid publication on acceptance

- support for research data, including large and complex data types

- gold Open Access which fosters wider collaboration and increased citations

- maximum visibility for your research: over $100 \mathrm{M}$ website views per year

At BMC, research is always in progress.

Learn more biomedcentral.com/submissions 